\title{
A CRITICAL INVESTIGATION OF VARIOUS SUBCLASSES OF FUNCTIONS WHOSE REAL PART IS BOUNDED
}

\author{
S.K. BAJPAI \\ Universidade de Brasilia \\ Departamento de Matematica \\ 70.910 - Brasilia - DF \\ Brasil \\ (Recefved October 22, 1979)
}

ABSTRACT. In this paper we investigate various subclasses of univalent analytic functions. We find that many of the subclasses introduced in the recent years are no more new and infact coincide with the class due to Jakubowski. We further study the generalised Jakubowski class of univalent functions and obtain a representational formula and use it in deriving the coefficient relations for this class.

KEY WORDS AND PHRASES. Univalent Starlike, convex, Spiral like, Bounded functions, Functions with positive real part.

1980 MATHEMATICS SUBJECT CLASSIFICATION CODES. Primary 30A32, Secondary $30 A 34$.

1. INTRODUCTION.

The class $s_{m, M}^{*}(\mu, \nu)$.

Let $M, m$ be arbitrary fixed real numbers satisfying the condition 
$(m, M) \varepsilon D^{*}=D_{1} \cup D_{2}$ and

$$
\begin{aligned}
& D_{1}=\left\{(m, M) \mid \frac{1}{2}<m<1,1-m<M \leq m\right\} \\
& D_{2}=\{(m, M) \mid m \geq 1, \quad m-1 \leq M \leq m\}
\end{aligned}
$$

and also $\mu, \nu$ be real numbers such that $0<\mu<1$ and $|\nu| \leq \frac{\pi}{2}$. In 1971, Jakubowski [7] introduced the class $s_{m, M}^{*}(\mu, \nu)$ of regular functions $f(z)=$ $z+\sum_{k=2}^{\infty} a_{k} z^{k}$ defined in $E=\{z|| z \mid<1\}$ and satisfying the condition

$$
\left|\frac{\frac{e^{i \nu} z f^{\prime}(z)}{f(z)}-i \sin \nu-\mu \cos \nu}{(1-\mu) \cos \nu}-m\right|<M,
$$

for all $z \in E$.

Recently, Mogra and Juneja ([9], [18], [17]) introduced a new class of starlike functions of order $\alpha$ and type $\beta$ which they denoted by $s^{\star}(\alpha, \beta)$. Let $S=\left\{f \mid f(z)=z+\sum_{k=2}^{\infty} a_{k} z^{k}\right.$ and $f$ is analytic in $\left.E\right\}$. They put $f \in S$ in $S^{*}(\alpha, \beta)$ if and only if

$$
1>\left|\left(\frac{z f^{\prime}(z)}{f(z)}-1\right) /\left\{2 \beta\left(\frac{z f^{\prime}(z)}{f(z)}-\alpha\right)-\left(\frac{z f^{\prime}(z)}{f(z)}-1\right)\right\}\right|,
$$

for all $\alpha, \beta$ real, $0 \leq \alpha<1,0<\beta \leq 1$.

Mogra and Juneja proposed that this class enables simple definition, and that thinking of this class helps in studying, other classes of starlike functions. Lakshminarasimhan [13] introduced the class $M(\alpha, \beta)$, containing $f \varepsilon S$ with

$$
\left|\frac{z f^{\prime}(z)}{f(z)}-1\right|<\beta \mid \alpha^{z f^{\prime}(z)} \frac{f(z)}{f(z \mid}
$$

for $0 \leq \alpha<1,0<\beta \leq 1$ and $z \in E$.

Mokowka $[19]$ generalized $s^{*}(\alpha, \beta)$ into $s^{\lambda}(\alpha, \beta)$ containing, for $|\lambda|<\frac{\pi}{2}$, those $f \varepsilon S$ with 


$$
\left|\frac{z f^{\prime}(z)}{f(z)}-1\right|<\beta\left|\frac{z f^{\prime}(z)}{f(z)}-1+2(1-\alpha) \cos \lambda e^{-i \lambda}\right|
$$

Yet another class is investigated by Gopalakrishna and Shetiya [6]. They named this class by $s_{\lambda}(\alpha, \rho, \eta)$ and $f \in s_{\lambda}(\alpha, \rho, \eta)$ if and only if $f \in s$ with

$$
\left|\frac{\frac{e^{i \lambda} z f^{\prime}(z)}{f(z)}-i \sin \lambda}{\cos \lambda}-\alpha-\rho\right|<\rho,
$$

for $|\lambda|<\frac{\pi}{2}, 0 \leq \alpha<1$ and $\alpha+\rho \geq 1$.

But we now show that these classes are not new and some of the results of the above authors are also not new. In fact, we have

THE OREM 1 .

(a) $s^{*}(\alpha, \beta)=s_{M, M}^{*}(\alpha, 0) ; M=1 / 2(1-\beta)$

(b) $s^{\lambda}(\alpha, \beta)=s_{1+M, M}^{*}(\alpha, \lambda) ; M=2 \beta^{2} /\left(1-\beta^{2}\right)$

(c) $M(\alpha, \beta)=s_{m, M}^{*}(0,0) ; m=\left(1+\alpha \beta^{2}\right) /\left(1-\alpha^{2} \beta^{2}\right)$ and $M=(1+\alpha) \beta /\left(1-\alpha^{2} \beta^{2}\right)$

(d) $s_{\lambda}(\alpha, \rho, n)=s_{M, M}^{*}(\alpha, \lambda) ; M=\rho /(1-\alpha)$.

PROOF. Suppose $X=z f^{\prime}(z) / f(z)$. Then $f \varepsilon s^{*}(\alpha, \beta)$ satisfies (1.2). Hence, we have

$$
|(x-1) /\{2 \beta(x-\alpha)-(x-1)\}|<1
$$

The inequality (1.6) is true if and only if

$$
\beta|x-\alpha|^{2}>\operatorname{Re}\{(x-\alpha)(\bar{x}-1)\} \text {. }
$$

This last inequality is equivalent to

$$
|x|^{2}-\left\{\frac{1+\alpha-2 \alpha \beta}{1-\beta}\right\} \operatorname{Re}\{x\}+\frac{\alpha(1-\alpha \beta)}{(1-\beta)}<0 .
$$

By simple manipulations, the last inequality holds if and only if 


$$
\left|\frac{\left(\frac{z f^{\prime}(z)}{f(z)}-\alpha\right)}{1-\alpha}-\frac{1}{2(1-\beta)}\right|<\frac{1}{2(1-\beta)} .
$$

Hence, if $M=\frac{1}{2(1-\beta)}, \mu=\alpha$ with $\nu=0$ then $f \in s^{*}(\alpha, \beta)$ implies $f \in s_{M, M}^{*}(\alpha, 0)$ and conversely. This proves (a).

For proving (b), we let $Y=\left\{X e^{i \lambda}-i \sin \lambda-\alpha \cos \lambda\right\} /(1-\alpha) \cos \lambda$ and $x=\frac{z f^{\prime}(z)}{f(z)}$. Then, sefore $f \in s^{\lambda}(\alpha, \beta)$ if and only if

$$
|Y-1|<\beta|Y+1| \text {. }
$$

(In (1.8), if $\lambda=0=\alpha$, then this class is due to Caplinger and Causey [3]). But simple calculations show that (1.8) is equivalent to

$$
\left|\frac{e^{i \lambda} \frac{z f^{\prime}(z)}{f(z)}-i \sin \lambda-\alpha \cos \lambda}{(1-\alpha) \cos \lambda}-1-\frac{2 \beta^{2}}{1-\beta^{2}}\right|<\frac{2 \beta^{2}}{1-\beta^{2}} .
$$

Thus, a comparision of (1.9) to (1.1) implies (b) and conversely. The proof for (c) is similar. For (d) we observe that $f \varepsilon s_{\lambda}(\alpha, \rho, \eta)$ if and only if

$$
\left|\frac{\frac{e^{i \lambda} z f^{\prime}(z)}{f(z)}-i \sin \lambda-\alpha \cos \lambda}{(1-\alpha) \cos \lambda}-\frac{\rho}{1-\alpha}\right|<\frac{\rho}{1-\alpha} \text {. }
$$

Again a comparision of (1.10) to (1.1) gives (d). Hence the proof of the theorem is complete.

We note a consequence of theorem 1, that the coefficients estimates obtained in the series of papers $([1]-[6],[8]-[11],[13]-[27],[29],[30])$ are contained in [7]. But some of the coefficients estimates obtained in [18], [6], [12] are not contained in the theorem 1 of [7]. We also note that the class introduced in [28], is a very general one but appears unnatural in the presentation. Therefore, next section, we shall consider the natural generalization of the class $s_{m, M}^{*}(\mu, \nu)$ by admitting $m$ to be complex. This we do as follows. 
2. The class $s_{m, M}^{*}(\mu, v, \alpha, t)$.

We define the class $s_{m, M}^{*}(\mu, \nu, \alpha, t)$ to denote the class of $f \varepsilon s$ with

$$
\left|\frac{\frac{e^{i \nu} z f^{\prime}(z)}{f(z)}-i \sin \nu-\mu \cos \nu}{(1-\mu) \cos \nu}-m-\alpha-i t\right|<M,
$$

where $m, \alpha, t$ and $M$ are real numbers such that $\frac{-\pi}{2}<\nu<\frac{\pi}{2}, \mu<1, \frac{1}{2}<m$, $0<M,-\infty<\alpha<1,-\infty<t<\infty$ and $M^{2}=1+2 m+2 \alpha-2 m \alpha-m^{2}-\alpha^{2}-t^{2}>0$.

The following lemma shows that when $\nu=0$, the utmost class of Szynal et al [28] and the class introduced by Libera and Livingston [21] are obtained.

LEMMA 1. $f \in S_{m, M}^{*}(\mu, v, \alpha, t)$ if and only if the re exists some $\omega(z)$ regular in $E, \omega(0)=0$ and $|\omega(z)|<1$ such that

$$
\frac{z f^{\prime}(z)}{f(z)}=\frac{1+\left(D e^{-i \nu}-B\right) \omega(z)}{1-B \omega(z)}
$$

where $D=(A+B)(1-\mu) \cos \nu ; A+B>0$

and

$$
A=\frac{1}{M}\left[M^{2}-m^{2}+m(1-2 \alpha)+\alpha(1-\alpha)+i t-t^{2}\right]
$$

$$
B=\frac{1}{M}[-1+m+\alpha-i t] \text {. }
$$

PROOF. Let us write

$$
\begin{aligned}
& L(z)=\frac{1}{\bar{M}}\left[\frac{g(z)}{h(z)}-m-\alpha-i t\right] \\
& g(z)=e^{i \nu} z f^{\prime}(z)-i \sin \nu f(z)-\mu \cos \nu f(z)
\end{aligned}
$$

and

$$
h(z)=(1-\mu) \cos \nu f(z) \text {. }
$$

Then, we have $|L(z)|<1, L(0)=\frac{1}{M}[1-m-\alpha-i t]$. Hence, there exists a $\omega(z)$ which is regular in $E$ and which can be written in the form 


$$
\omega(z)=\frac{L(z)-L(0)}{1-\overline{L(0) L(z)}} .
$$

Clearly, we have $\omega(0)=0$ and $|\omega(z)|<1$. Solving the equation (2.3), we have

$$
L(z)=\frac{1}{M}\left[\frac{g(z)}{h(z)}-m-\alpha-i t\right]=\frac{L(0)+\omega(z)}{1+\overline{L(0)} \omega(z)},
$$

and this in turn on solving gives the lemma.

Using this lemma, we prove the following theorem which generalizes the results in [28], [12] and [6].

THEOREM 2. For $|z|<1$, let $f(z)=z+\sum_{k=n+1}^{\infty} a_{k} z^{k}$ and $f \varepsilon s_{m, M}(\mu, v, \alpha, t)$. Also, let $q_{0}$ be a natural number such that $q_{0} \varepsilon\left[k_{0}-1, k_{0}\right)$ where $K_{0}=D\left[\operatorname{Re}\left\{B e^{i \nu}\right\}+\sqrt{1-|B|^{2}+\left(\operatorname{Re}\left\{B e^{i \nu}\right\}\right)^{2}}\right]\left\{n\left(1-|B|^{2}\right)\right\}^{-1}$. Then

(i) If, either $\operatorname{Re}\left\{B e^{i \nu}\right\} \geq 0$ and $M(1-\mu) \cos \nu\left[D+2 n \operatorname{Re}\left\{B e^{i \nu}\right\}\right] \leq n^{2}$ or, $\operatorname{Re}\left\{B e^{i \nu}\right\} \leq 0$ and $\operatorname{DM}(1-\mu) \cos \nu \leq \mathrm{n}^{2}$ then we have

$$
\sum_{k=n p+1}^{(p+1) n}(k-1)^{2}\left|a_{k}\right|^{2} \leq D^{2} ; p=1,2,3, \ldots
$$

(ii) If $\operatorname{Re}\left\{B e^{i \nu}\right\}>0$ and $(1-\mu) M \cos \nu\left[D+2 n \operatorname{Re}\left\{B e^{i \nu}\right\}\right]>n^{2}$ then we have

$$
\sum_{k=q n+1}^{(q+1) n}(k-1)^{2}\left|a_{k}\right|^{2} \leq\left\{\frac{n}{(q-1) !} \prod_{m=0}^{q-1}\left|\frac{p+m n B e^{i \nu}}{n}\right|\right\}^{2},
$$

for $q=1,2, \ldots, q_{0}+1$ and $q n+1 \leq k \leq(q+1) n$.

(iii) If $\operatorname{Re}\left\{B e^{i \nu}\right\}>0$ and $(1-\mu) M \cos \nu\left[D+2 n \operatorname{Re}\left\{B e^{i \nu}\right\}\right]>n^{2}$ then for $q=q_{0}+2, \ldots$, we have

$$
\sum_{k=q n+1}^{(q+1) n}(k-1)^{2}\left|q_{k}\right|^{2} \leq\left\{\left(\frac{n}{q_{0}}\right) \prod_{m=0}^{q}\left|\frac{D+m n B e^{i \nu}}{n}\right|\right\}^{2},
$$

for $q n+1 \leq k \leq(q+1) n$. 
The estimates (2.4) are sharp for all $k$ and the estimates in (2.5) are sharp for $k=q n+1, q=1,2, \ldots$ because equality holds for the functions

$$
f(z)= \begin{cases}\frac{z}{\left(1-B z^{k}\right) D e^{-i v / k B}} & \text { for } B \neq 0 \\ z e^{D e^{-i \nu / k / k}} & \text { for } B=0\end{cases}
$$

PROOF. Under the conditions of theorem 2, we have

$$
\omega(z)=\sum_{k=n}^{\infty} b_{k} z^{k}
$$

for which (2.2) is true. Then simple calculations lead to

$$
e^{i v} \sum_{k=n+1}^{\infty} a_{k}(k-1) z^{k}=\left[D z+\sum_{k=n+1}^{\infty}\left\{D+B(k-1) e^{i \nu}\right\} a_{k} z^{k}\right]\left[\sum_{k=n}^{\infty} b_{k} z^{k}\right] .
$$

Equating the coefficients of $z^{k}$ from both sides of $(2.7)$ for $k=n+1, \ldots, 2 n$, we have

$$
e^{i \nu}(k-1) a_{k}=D b_{k-1}
$$

This immediately gives us,

$$
\sum_{k=n+1}^{2 n}(k-1)^{2}\left|a_{k}\right|^{2} \leq D^{2} \sum_{k=n+1}^{\infty}\left|b_{k}\right|^{2} \leq D^{2} .
$$

But, for $p \geq n+1,(2.7)$ can be written in the form

$$
G(z)=H(z) \omega(z),
$$

where

$$
G(z)=e^{i \nu} \sum_{k=n+1}^{n+p}(k-1) a_{k} z^{k}+\sum_{k=n+p+1}^{\infty} d_{k} z^{k}
$$

and

$$
H(z)=D z+\sum_{k=n+1}^{p}\left\{D+B(k-1) e^{i \nu}\right\} a_{k} z^{k} .
$$

Then (2.10) gives us 


$$
\begin{array}{r}
\sum_{k=p+1}^{n+p}(k-1)^{2}\left|a_{k}\right|^{2}+\sum_{k=n+p+1}^{\infty}\left|d_{k}\right|^{2} \\
\leq D^{2}+\sum_{k=n+1}^{p}\left\{\left|D+(k-1) B e^{i \nu}\right|^{2}-(k-1)^{2}\right\}\left|a_{k}\right|^{2} .
\end{array}
$$

From the hypothesis of (i) of theorem 2, we have

$$
\left|\mathrm{D}+(\mathrm{k}-1) B \mathrm{Be}^{\mathrm{i} v}\right|^{2} \leq(\mathrm{k}-1)^{2}
$$

and so

$$
\sum_{k=p+1}^{p+n}(k-1)^{2}\left|a_{k}\right|^{2} \leq D^{2} ; \quad p \geq n+1
$$

Replacing $p$ by pn in (2.12) then we have

$$
\sum_{k=p n+1}^{(p+1) n}(k-1)^{2}\left|a_{k}\right|^{2} \leq D^{2} \text { for } p=2,3, \ldots
$$

Combining (2.13) with $(2.9)$, we get (i).

If $\operatorname{Re}\left\{B e^{i \nu}\right\}>0$ then the function

$$
\left(\frac{1}{k-1}\right)^{2}\left[\left|D+(k-1) B e^{i \nu}\right|^{2}-(k-1)^{2}\right]
$$

is always a decreasing function of $k-1$.

On using this fact, we get, by $(2.11)$ for $p \geq n+1$.

$$
\sum_{k=p+1}^{p+n}(k-1)^{2}\left|a_{k}\right|^{2} \leq D^{2}+\sum_{k=n+1}^{p}\left\{\frac{\left|D+n B e^{i \nu}\right|^{2}-n^{2}}{n^{2}}\right\}(k-1)^{2}\left|a_{k}\right|^{2} .
$$

Let $p=2 n$. Then (2.14) gives us

$$
\sum_{2 n+1}^{(2+1) n}(k-1)^{2}\left|a_{k}\right|^{2} \leq\left\{D\left|\frac{D+B n e^{i \nu}}{n}\right|\right\}^{2} \text { by (2.9), }
$$

Under the conditions of (ii) of theorem 2, we intend to prove the following.

$$
\sum_{k=q n+1}^{(q+1) n}(k-1)^{2}\left|a_{k}\right|^{2} \leq\left\{\frac{n}{(q-1) !} \prod_{m=0}^{q-1}\left|\frac{D+m n B e^{i \nu}}{n}\right|\right\}^{2} .
$$


The relation (2.16) is true for $q=1$ and 2 , so let us assume (2.16) to be true for $1,2, \ldots, q-1$ and let us prove it for $q$ if $q \leq q_{0}+1$. By (2.14) we have

$$
\begin{aligned}
& \sum_{k=q n+1}^{(q+1) n}\left|a_{k}\right|^{2}(k-1)^{2} \leq D^{2}+\sum_{k=n+1}^{q n}\left\{\left|D+(k-1) B e^{i \nu}\right|^{2}-(k-1)^{2}\right\}\left|a_{k}\right|^{2} \\
& =D^{2}+\sum_{m=1}^{q-1} \sum_{k=m n+1}^{(m+1) n}\left\{\frac{\left|D+(k-1) B e^{i \nu}\right|^{2}-(k-1)^{2}}{(k-1)^{2}}\right\}(k-1)^{2}\left|a_{k}\right|^{2} \\
& \leq D^{2}+\sum_{m=1}^{q-1}\left\{\frac{\left|D+B m n e^{i \nu}\right|^{2}-m^{2} n^{2}}{m^{2} n^{2}}\right\}\left\{\left(\frac{n}{(m-1) !}\right)_{m=0}^{m-1}\left|\frac{D+m B e^{i \nu}}{n}\right|\right\}^{2} \\
& =\left\{\frac{n}{(q-1) !} \prod_{m=0}^{q-1} \mid \frac{D+m n B e^{i \nu}}{n}\right\}^{2}
\end{aligned}
$$

The last inequality also follows by induction. This proves (ii) of theorem 2 . Also, if $k>\left(q_{0}+2\right) n$ then

$$
\begin{aligned}
& \left.\sum_{k=q n+1}^{(q+1) n}(k-1)^{2}\left|a_{k}\right|^{2} \leq D^{2}+\left[\int_{k=n+1}^{\left(q_{0}+1\right) n}+\sum_{k=\left(q_{0}^{+1) n+1}\right.}^{q n}\right\}\left\{\left|D+B e^{i \nu}(k-1)\right|^{2}-(k-1)^{2}\right\}\left|a_{k}\right|^{2}\right] \\
& \leq D^{2}+\sum_{k=n+1}^{(q+1) n}\left\{\frac{\left|D+B e^{i \nu}(k-1)\right|^{2}-(k-1)^{2}}{(k-1)^{2}}\right\}(k-1)^{2}\left|a_{k}\right|^{2} \\
& =D^{2}+\sum_{m=1}^{q} \sum_{k=m n+1}^{(m+1) n}\left\{\frac{\left|D+B e^{i \nu}(k-1)\right|^{2}-(k-1)^{2}}{(k-1)^{2}}\right\}(k-1)^{2}\left|a_{k}\right|^{2} \\
& \leq\left\{\frac{n}{q_{0}} \prod_{m=0}^{q}\left|\frac{D+m n B e^{i \nu}}{n}\right|\right\}^{2} .
\end{aligned}
$$

This proves (iii) of theorem 2. Hence the proof of the theorem is complete.

COROLLARY. Fram (2.11) we obtain

$$
\sum_{k=n+1}^{p}\left\{(k-1)^{2}-\left|D+(k-1) B e^{i \nu}\right|^{2}\right\}\left|a_{k}\right|^{2} \leq D^{2} .
$$

Our theorem 2 generalizes the results of [6], [28], [12], [7] and hence the results obtained in ([1]-[27]) are contained in theorem 2 for the different choices of the parameters, $m, M, \mu, \nu, \alpha$ and $t$. 


\section{REFERENCES}

[1] BAJPAI, S.K. and Mehrok,T.J.S.: On the coefficient structure and a growth theorem for the functions $f(z)$ for which $z f^{\prime}(z)$ is spirallike, Publ. De L'Institute Mathematique 16 (30), (1973), 5-12.

[2] BOY, A.V.: Coefficients estimates for starlike functions of order $\alpha$. Proc. Amer. Math. Soc. 17 (1966), 1016-1018.

[3] CAPLINGER, T.R. and Causey, W.M.: A class of univalent functions, Proc. Amer. Math. Soc. 39 (1973), 357-361.

[4] CLUNIE, J. and Keogh, F.R.: On starlike and convex schlicht functions. J. London Math. Soc. 35 (1960), 229-233.

[5] EENIGENBURG, P.J.: A class of starlike mappings in the unit dis $\mathrm{C}$, composition Math 24 (1972), 235-238.

[6] GOPALAKRISHNA, H.S. and Shetiya,V.S.: Coefficient estimates for spiral like functions. Annales Polon Math. 35 (1977), 1-9.

[7] JAKUBOWSKI, Z.J.: On the coefficients of starlike functions of some classes. Bu11. Acad. Polon. des Sci., Serie des Sci. Math., Ast. et phys. 19 (1971), 811-815.

[8] JANOWSKI, W.: About the extremal spiral schlicht functions, Annales Polon. Math: 9 (1962), 265-273.

[9] JUNEJA,O.P. and Mogra,M.L.: On starlike functions of order $\alpha$ and type $\beta$. Kev. Roum. Math. 23 (1978), 751-765.

[10] KACZMARSKI, J.: On the coefficients of some classes of starlike functions Bu11. Acad. Polon. Sci. Sér. Sci. Math., ast. Phys. 17 (1969), 495-501.

[11] KLEIN,M .: Functions starlike of order $\alpha$. Trans Amer. Math. Soc. 131(1), (1968), 99-106.

[12] LIBERA, R.J. and Livingston,A.E.: Bounded functions with positive real part. Czechos lov ak Math. J. 22(97), (1972).

[13] LAKSHMINARASIMHAN, T.V.: On sub-classes of functions starlike in the unit disc. J. Indian Math. Soc. 41 (1977), 233-243.

[14] LIBERA, R.J.: Univalent $\alpha$-spiral functions. Canadian J. Math. 19 (1967), 449-456.

[15] MACGREGOR, T.H.: Coefficient estimates for starlike mappings. Michigan Math. J. 10 (1963), 277-281.

[16] MCCARTY, C.P.: Starlike functions. Proc. Amer. Math. Soc. 43 (1974), 361-366.

[17] MOGRA, M.L.: On a subclass of starlike functions in the unit disc. J. Indian Math. Soc. 40 (1976). 
[18] MOGRA, M.L. and Juneja, O.P.: Coefficient estimates for starlike functions, Bul1. Aust. Math. Soc. 16 (1977), 415-425.

[19] MOKOWKA, B.: On some subclasses of univalent functions; Politechn. Lodz, Zeszyty Nank 254, Mat $\underline{9}$ (1977), 71-76.

[20] NEVANLINA, R.: Uber die Konforme abbildung von sterngebieten, Ofvers Finska Vet. Ser. Forh, $\underline{53(A)}(1921), \underline{6}$.

[21] PADMANABHAN, K.S.: On certain classes of starlike fun dions in the unit disc. J. Indian Math. Soc. 32 (1968), 89-103.

[22] PLASKOTA, W.: On the coefficients of some families of regular functions, Bul1. Acad. Polon. Sci. Sér. Sci. Math. ast. phys. 17 (1969), 715-718.

[23] PLASKOTA, W.: Limitation des coefficients dans une famille de functions holom-orphes dans de cercle $|z|<1$. Annales Polon Math. Soc. 24 (1970), 65-70.

[24] ROBERTSON, M.S.: On the theory of univalent functions, Ann. of Math. 37 $(1936), 374-408$.

[25] SCHIL, A.: On a class of univalent star shaped mappings. Proc. Amer. Math. Soc. $\underline{9}$ (1958), 751-757.

[26] SCHILD, A.: On starlike functions of order $\alpha$. Amer J. Math. 87 (1965), 65-70.

[27] SINGH, RAM: On a class of starlike functions, Compositio Math. 19 (1967), 78-82.

[28] SZYNAL, A.; Szynal, J and Zygmunt, J.: On the coefficients of functions whose real part is bounded. Annales Univ. Mariae Curie-Sklod. Lubin Polon. 26 (5) (1972), 63-69.

[29] TONTI, N.E. and $\operatorname{Tr} a h a n, D . D .:$ Analytic functions whose real parts are bounded below. Math. $\underline{\text { Z. }} \underline{115}$ (1970), 252-258.

[30] WRIGHT, D.J.: On a class of starlike function. Compositio Math. 21 (1969), 122-124. 


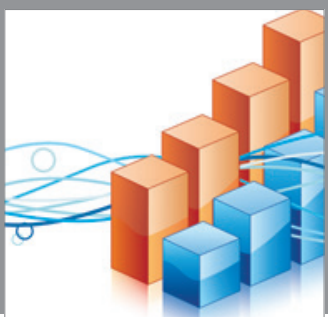

Advances in

Operations Research

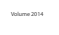

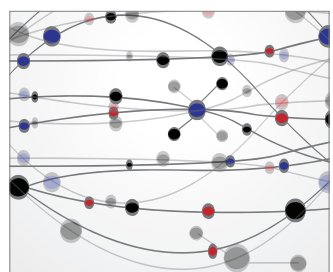

\section{The Scientific} World Journal
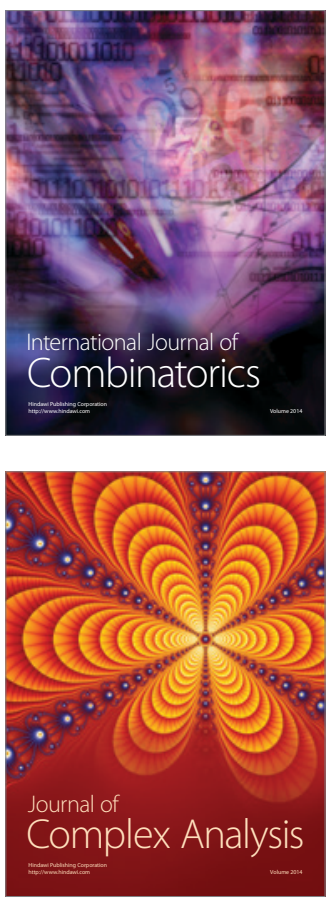

International Journal of

Mathematics and

Mathematical

Sciences
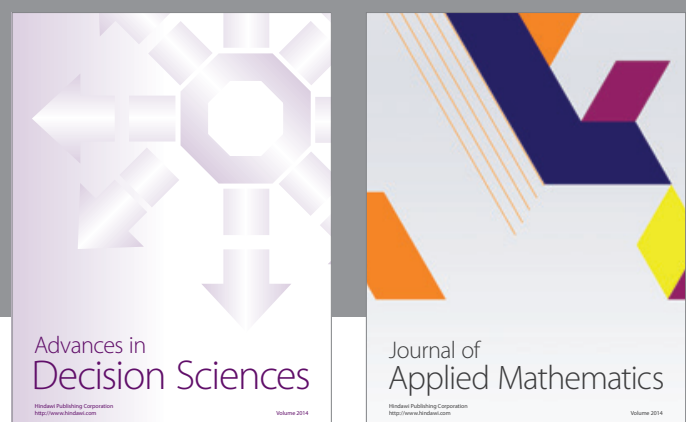

Journal of

Applied Mathematics
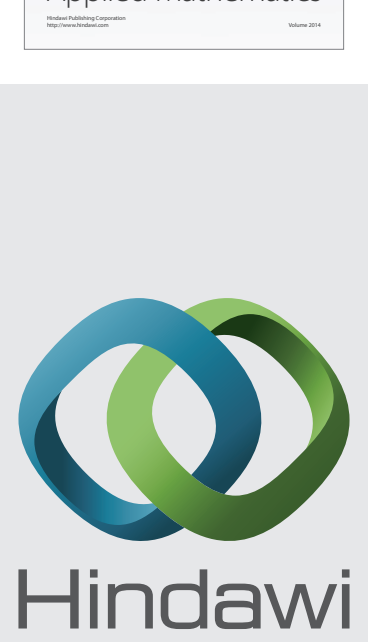

Submit your manuscripts at http://www.hindawi.com
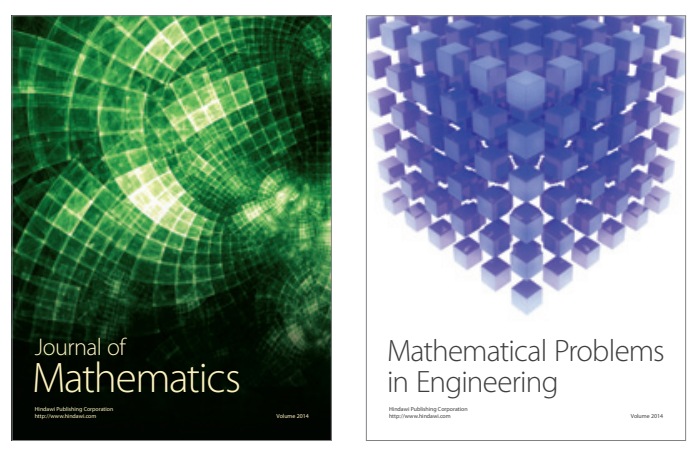

Mathematical Problems in Engineering
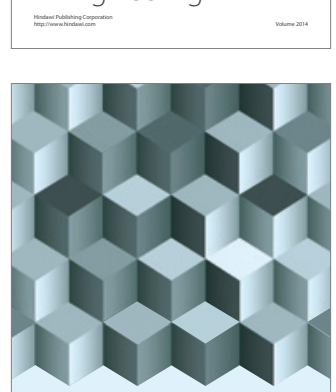

Journal of

Function Spaces
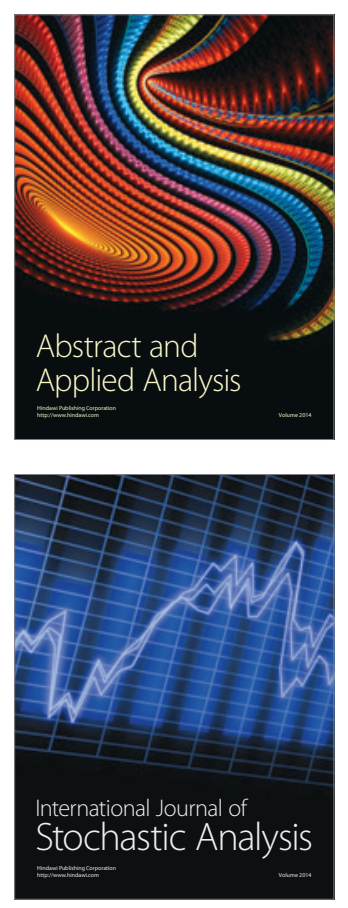

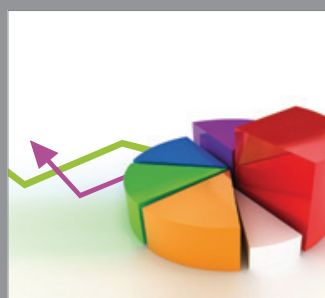

ournal of

Probability and Statistics

Promensencen
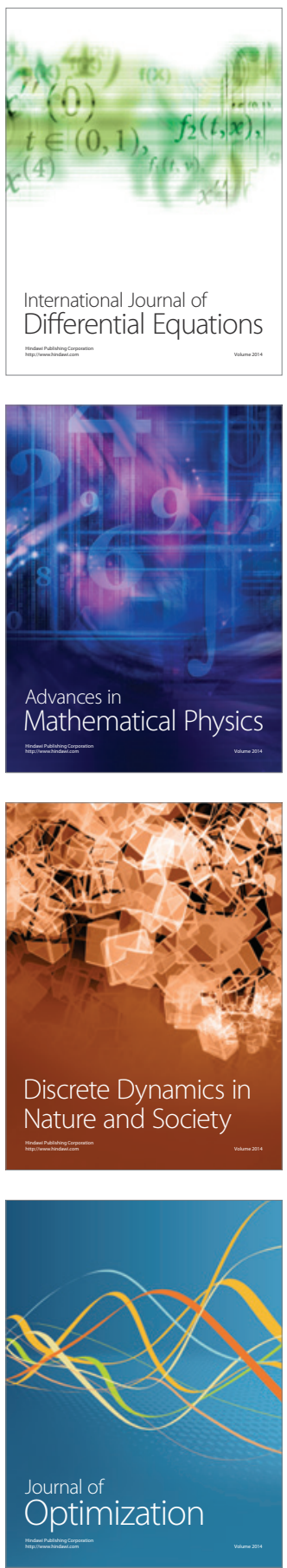